\title{
Multiplex loop-mediated isothermal amplification-based lateral flow dipstick for simultaneous detection of 3 food-borne pathogens in powdered infant formula
}

\author{
Yujun Jiang, ${ }^{1 *} \odot$ Sihan Chen, ${ }^{1 *}$ (1) Yueming Zhao, ${ }^{1}$ Xinyan Yang, ${ }^{1}$ Shiqian Fu, ${ }^{1}$ John L. McKillip, ${ }^{2} \odot$ \\ Edward M. Fox, ${ }^{3} \odot$ and Chaoxin $\operatorname{Man}^{1} \dagger$ - \\ ${ }^{1}$ Key Laboratory of Dairy Science, Ministry of Education, Department of Food Science, Northeast Agricultural University, Harbin, China, 150000 \\ ${ }^{2}$ Department of Biology, Ball State University, Muncie, IN 47303 \\ ${ }^{3}$ Department of Applied Sciences, Northumbria University, Newcastle upon Tyne, NE1 8ST United Kingdom
}

\section{ABSTRACT}

In this study, we established a rapid, simple, and sensitive method for visual and point-of-care detection of Salmonella spp., Cronobacter spp., and Staphylococcus aureus in powdered infant formula (PIF) based on multiplex loop-mediated isothermal amplification (mLAMP) combined with lateral flow dipstick (LFD). Three different species-specific target genes, siiA of Salmonella spp., internal transcribed space (ITS) of Cronobacter spp., and nuc of Staph. aureus, were applied in the mLAMP with biotin-, digoxin-, and Texas Red-modified forward inner primers and fluorescein isothiocyanate (FITC)-modified backward inner primers. After mLAMP, a large number of modified amplicons were detected with LFD; one end of the amplicon was conjugated to the anti-FITC antibody on gold nanoparticles and the other end to streptavidin (anti-digoxin or anti-Texas Red antibody) on test lines. Visual inspection of the device relies on the presence of a red band formed by accumulation of sandwich composites. The detection limits of this mLAMP-LFD assay for Salmonella spp., Cronobacter spp., and Staph. aureus in PIF without enrichment were $4.2,2.6$, and $3.4 \mathrm{cfu} / \mathrm{g}$, respectively. The whole method can be completed in less than $1 \mathrm{~h}$. Thus, mLAMP-LFD is a rapid and efficient method for simultaneously detecting Salmonella spp., Cronobacter spp., and Staph. aureus in PIF.

Key words: multiplex loop-mediated isothermal amplification, lateral flow dipstick, food-borne pathogen, powdered infant formula

Received September 3, 2019.

Accepted December 25, 2019.

*These authors contributed equally to this work.

†Corresponding author: mcxwh2006@qq.com

\section{INTRODUCTION}

As a significant burden on public health, food-borne pathogens cause large numbers of outbreaks worldwide. Food-borne illnesses are frequently reported to be caused by Salmonella spp. (Angulo et al., 2008; Hyeon et al., 2010), Cronobacter spp. (Drudy et al., 2006), and Staphylococcus aureus (Wang et al., 2012b). These pathogens infect newborns and infants via intrinsic or extrinsic contamination of reconstituted powdered infant formula (PIF), which represents a significant hazard to the health of infants. Illnesses include diarrhea (Hyeon et al., 2010), neonatal meningitis (Liu et al., 2006b), and food poisoning (Wang et al., 2012b). Hence, there is an urgent need to develop a highly sensitive platform for simultaneous detection of these 3 pathogens to monitor PIF and prevent associated infections.

Conventional detection methods to identify foodborne pathogens such as Salmonella spp., Cronobacter spp., and Staph. aureus typically rely on culture-based assays, which require 5 to $7 \mathrm{~d}$ for the processes of preenrichment, growth on selective media, and biochemical confirmation (Salmonella spp. additionally needs serological testing; Law et al., 2015). These detection methods are both time consuming and laborious (Shao et al., 2011). Although more recent methods using PCR or real-time PCR have been developed, they often require long detection times and expensive instruments, which limit their application in the field of rapid detection. Loop-mediated isothermal amplification (LAMP) is based on nucleic acid amplification and was developed by Notomi et al. (2000). The target fragments can be amplified by LAMP with high efficiency and satisfactory specificity, only using a heating block or heating water bath. Therefore, it is suitable for food safety testing or clinical diagnostics, especially in resource-poor environments or settings (Dhama et al., 2014; Kokkinos et al., 2014). 
Recently, the multiplex loop-mediated isothermal amplification (mLAMP) assay, which mixes several sets of primers in a single reaction system, has attracted attention. Unlike a single LAMP, mLAMP produces a more complex ladder-like pattern of amplicons, which cannot be analyzed by agarose gel electrophoresis (AGE). Several methods have been reported to detect and differentiate mLAMP products, such as real-time turbidity measurement (Wang et al., 2012a; Nagamine et al., 2014), restriction patterns based on an endonuclease recognition site in the LAMP inner primers (Shao et al., 2011), fluorophore-labeled primers combined with an intercalator dye (Tanner et al., 2012), and sequence-based barcodes coupled with nicking endonuclease-mediated pyrosequencing (Liang et al., 2012). However, these methods are limited by the use of precision instruments, making them unsuitable for point-of-care testing. As a rapid, visual, and user-friendly detection method, the lateral flow dipstick (LFD) presents great potential for point-of-care testing (Liu et al., 2006a; Jung et al., 2015). Because the LFD method can discriminate a mixture of mLAMP amplicons, it has been used for the detection of toxins (Chen et al., 2016), pathogenic Leptospira (Nurul Najian et al., 2016), and Babesia (Yang et al., 2016). To the best of our knowledge, there are no reports of 3 pathogenic species being simultaneously detected by the mLAMPLFD method in a single reaction.

In this study, we developed a method based on mLAMP-LFD for the simultaneous detection of Salmonella spp., Cronobacter spp., and Staph. aureus in PIF, providing a simple, rapid, sensitive, and low-cost detection system for point-of-care testing in the dairy industry.

\section{MATERIALS AND METHODS}

\section{Reagents}

Bst 2.0 WarmStart DNA polymerase and $\mathrm{MgSO}_{4}$ were purchased from New England BioLabs (Ipswich, MA). Betaine was obtained from Sigma-Aldrich (St. Louis, MO). Deoxynucleoside triphosphates (dNTP) were purchased from TianGen Co. Ltd. (Beijing, China). The nitrocellulose membrane was obtained from Sartorius (Göttingen, Germany). The plastic backing, sample pad, conjugate pad, and absorption pad were purchased from Ahlstrom Co. Ltd. (Helsinki, Finland). The mouse anti-fluorescein isothiocyanate (FITC) monoclonal antibody was obtained from Santa Cruz Biotechnology Co. Ltd. (Shanghai, China). The rabbit anti-Texas Red polyclonal antibody was purchased from Thermo Fisher Scientific (San Jose, CA). The streptavidin, mouse anti-digoxin monoclonal antibody and gold chloride trihydrate were obtained from Sigma (St. Louis, MO). Goat anti-mouse secondary polyclonal antibody (IgG) was purchased from Bioss Co. Ltd. (Beijing, China). Sucrose and BSA were acquired from Sangon Biotech Co. Ltd. (Shanghai, China). All other reagents were of analytical grade. The LFD sprayer and automatic programmable cutter were acquired from Biodot (Irvine, CA) and Autokun Co. Ltd. (Hangzhou, China), respectively.

\section{Bacterial Strains and DNA Extraction}

A total of 42 bacterial strains, including Salmonella spp. $(\mathrm{n}=13)$, Cronobacter spp. $(\mathrm{n}=8)$, Staph. aureus $(\mathrm{n}=6)$, and other nontarget bacteria $(\mathrm{n}=15)$ were used for specificity testing of the mLAMP-LFD assay and are listed in Table 1. All bacteria were cultured overnight in Luria-Bertani (LB) broth at $37^{\circ} \mathrm{C}$ with shaking at $150 \mathrm{rpm}$. Salmonella Typhimurium ATCC 14028, Cronobacter sakazakii ATCC 29544, and Staph. aureus ATCC 13565 were used as positive controls, and nuclease-free water was used as the negative control. Genomic DNA samples were extracted from $1 \mathrm{~mL}$ of enrichment broth by using the Gen Elute Bacterial Genomic DNA Kit (Tiange, Beijing, China) and suspended in $100 \mu \mathrm{L}$ of Tris-EDTA buffer. Genomic DNA of bacteria was stored at $-20^{\circ} \mathrm{C}$ until use.

\section{Design of LAMP Primers}

According to previous studies (Ruan et al., 2013; Wang et al., 2017; Zhao et al., 2017), 3 conserved fragments from the siiA gene of Salmonella spp., the internal transcribed spacer (ITS) sequences of Cronobacter spp., and the nuc gene of Staph. aureus were selected as target sequences to design LAMP primers using Primer Explorer V4 software (http://primerexplorer.jp/ elamp4.0.0/index.html). Primers are shown in Table 2 and were synthesized by Invitrogen (Shanghai, China). The forward inner primers (FIP) targeting the siiA, ITS, and nuc genes were labeled with biotin, digoxin, and Texas Red at the $5^{\prime}$ end, and the backward inner primers (BIP) of the 3 sets were labeled with FITC at the $5^{\prime}$ end.

\section{mLAMP Reaction}

The multiplex LAMP reaction was performed in a $25-\mu \mathrm{L}$ volume containing the following components: 0.1 $\mu M$ siiA-F3 and siiA-B3 primers, $0.8 \mu M$ siiA-FIP and siiA-BIP primers, $0.1 \mu M$ ITS-F3 and ITS-B3 primers, $0.7 \mu M$ ITS-FIP and ITS-BIP primers, $0.1 \mu M$ nucF3 and nuc-B3 primers, $0.9 \mu M n u c-F I P$ and $n u c-B I P$ 
Table 1. Strains used for the multiplex loop-mediated isothermal amplification (mLAMP) specificity test $^{1}$

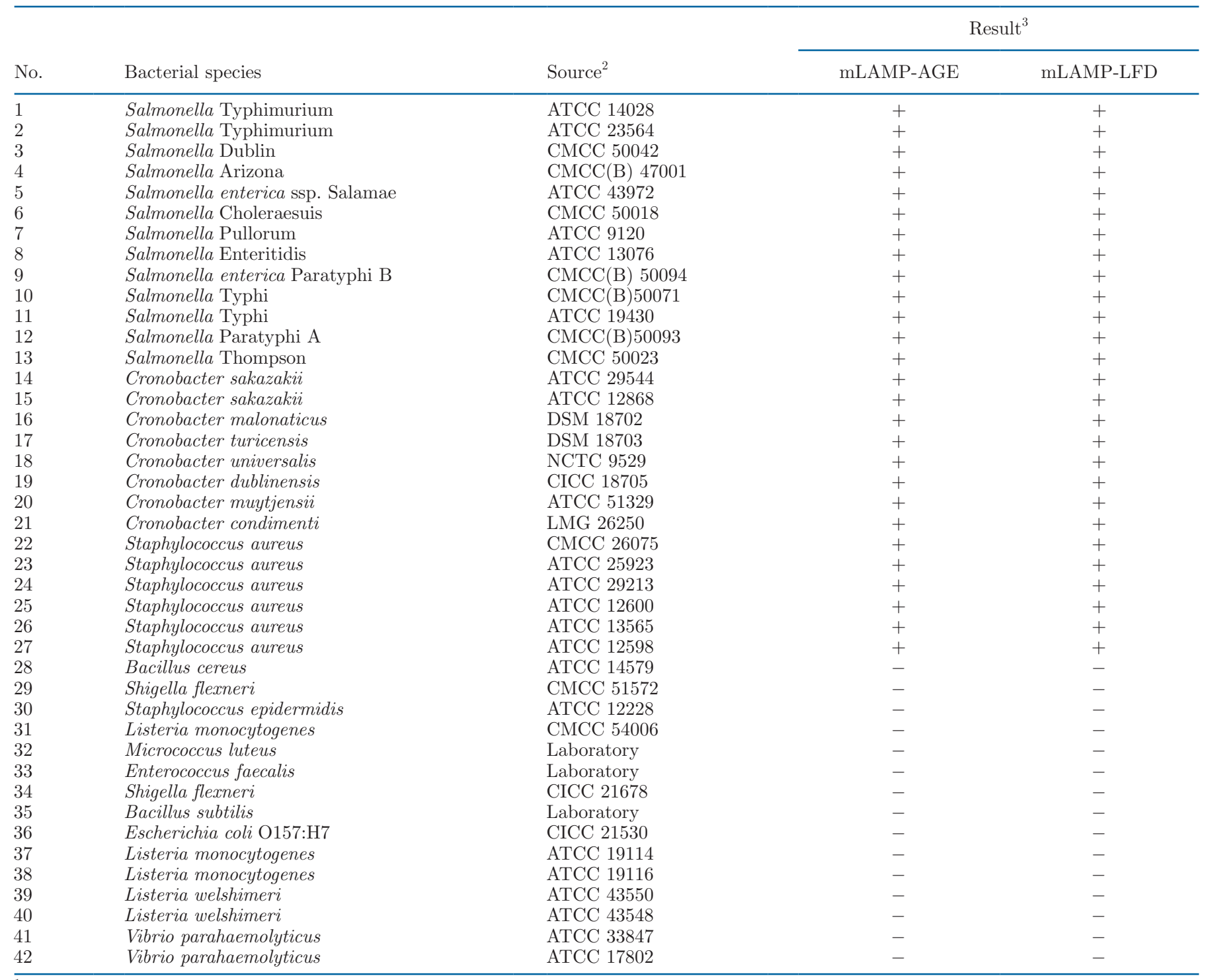

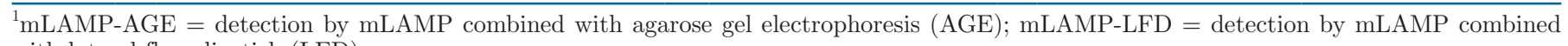
with lateral flow dipstick (LFD).

${ }^{2}$ ATCC = American Type Culture Collection (Manassas, VA); CMCC = China Medical Culture Collection Center, Ministry of Public Health (Beijing, China); DSM = DSMZ-Deutsche Sammlung von Mikroorganismen und Zellkulturen GmbH (Braunschweig, Germany); NCTC = National Collection of Type Cultures, Central Public Laboratory Service (London, UK); CICC = China Center of Industrial Culture Collection, China National Research Institute of Food and Fermentation Industries (Beijing, China); LMG = Laboratory of Microbiology, Department of Biochemistry and Microbiology, Faculty of Sciences of Ghent University (Ghent, Belgium).

${ }^{3}+$, positive result; - , negative result.

primers, $1.6 \mathrm{~m} M \mathrm{dNTP}, 4 \mathrm{~m} M \mathrm{MgSO}_{4}, 2.5 \mu \mathrm{L}$ of $10 \times$ ThermoPol Buffer (New England BioLabs), $0.9 \mu \mathrm{L}$ of $8 \mathrm{U} / \mu \mathrm{L}$ Bst 2.0 WarmStart DNA polymerase (New England BioLabs), and $2 \mu \mathrm{L}$ of template DNA (68.5 $\mathrm{ng} / \mu \mathrm{L}$ ) of each target bacteria. Then, amplification was carried out in a conventional water bath and incubated at $64^{\circ} \mathrm{C}$ for $45 \mathrm{~min}$ followed by $80^{\circ} \mathrm{C}$ for $2 \mathrm{~min}$ to terminate the reactions.

\section{Establishment of the LFD}

Preparation of Gold Nanoparticles and Functionalization with Antibody. Gold nanoparticles (AuNP), with a diameter of $15 \pm 3.5 \mathrm{~nm}$, were prepared based on a previously reported method (He et al., 2011; Chen et al., 2016). Three microliters of $0.2 \mathrm{M} \mathrm{K}_{2} \mathrm{CO}_{3}$ was added to $1 \mathrm{~mL}$ of AuNPs to adjust them to a suit- 
Table 2. Sequences of multiplex loop-mediated isothermal amplification (mLAMP) primers

\begin{tabular}{llc}
\hline Primer & Sequence $\left(5^{\prime} \rightarrow 3^{\prime}\right)$ & Label \\
\hline nuc-F3 & CGATTGATGGTGATACGGTTA & \\
nuc-B3 & CCACGTCCATATTTATCAGTTC & $5^{\prime}$-modified Texas Red \\
nuc-FIP & GGATGCTTTGTTCAGGTGTATCATGTACAAAGGTCAACCAATG & $5^{\prime}$-modified FITC \\
nuc-BIP & AGAGAAATATGGCCTGAAGCAAGCCTTTGTCAAACTCGACTTC & \\
ITS-F3 & AAATGCGCGGTGTGTCAG & \\
ITS-B3 & GGTTTCCCATTCGGACAT & $5^{\prime}$-modified digoxin \\
ITS-FIP & ACCGTGTACGCTTGTTCGCTTTCTCTCAAACTCGCAGCAC & $5^{\prime}$-modified FITC \\
ITS-BIP & GGCAGTCAGAGGCGATGCGCCGGTTATAACGGTTCA & \\
siiA-F3 & AGAAGCTTTTCAGAAGAGGA & \\
siiA-B3 & GATAATAGCTTTACTCGTTGTACT & $5^{\prime}$-modified biotin \\
siiA-FIP & CTGCCTGAGGATTACCATTTCTATAATAAAGGCTTGGTTAGAAGACA & $5^{\prime}$-modified FITC \\
siiA-BIP & TACGACTGGGATATGAACGGGACTCATGTCAATAACTACATCAG & \\
\hline
\end{tabular}

${ }^{1}$ Fluorescein isothiocyanate.

able $\mathrm{pH}$. Since AuNPs would damage the electrode of the $\mathrm{pH}$ meter, $0.2 \mathrm{M} \mathrm{K}_{2} \mathrm{CO}_{3}$ was added to the solution as a $\mathrm{pH}$ indicator. Then, $3 \mu \mathrm{L}$ of $1 \mathrm{mg} / \mathrm{mL}$ anti-FITC antibody was added to the solution and incubated at room temperature for $15 \mathrm{~min}$. Then, 10\% BSA (wt/vol) was added and mixed thoroughly, blocking the residual sites on the surface of AuNPs. After incubation for 15 min, the mixture was centrifuged at $9,175 \times g$ at $4^{\circ} \mathrm{C}$ for $15 \mathrm{~min}$. Subsequently, the supernatant was removed and the crimson precipitate was resuspended in $100 \mu \mathrm{L}$ of dilution buffer (wt/vol) containing $100 \mathrm{~m} M$ Tris ( $\mathrm{pH}$ 9), $4 \%$ trehalose (wt/vol), and $0.2 \%$ BSA and stored in $4^{\circ} \mathrm{C}$ until further use.

Assembly of the LFD. The LFD was assembled from 4 components (sample pad, conjugate pad, nitrocellulose membrane, and absorbent pad), which were pasted on the polyvinyl chloride (PVC) baseplate. The sample and conjugate pads were saturated with running buffer $[100 \mathrm{~m} M$ Tris (pH 9), $0.05 \%$ casein (wt/ vol), $0.5 \%$ Tween 20, $4 \%$ trehalose (wt/vol), $0.002 \%$ trisodium citrate (wt/vol), $1 \%$ polyvinylpyrrolidone (PVP; wt/vol), $0.2 \% \mathrm{BSA}$ (wt/vol), and $0.05 \%$ sodium azide (wt/vol)] and then dried at $45^{\circ} \mathrm{C}$ for $2 \mathrm{~h}$. Then, the conjugate pad was treated with anti-FITC-AuNP conjugates and dried at $37^{\circ} \mathrm{C}$ for $2 \mathrm{~h}$. Streptavidin $(0.8$ $\mathrm{mg} / \mathrm{mL})$, anti-digoxin antibody $(0.8 \mathrm{mg} / \mathrm{mL})$, antiTexas Red antibody $(0.8 \mathrm{mg} / \mathrm{mL})$, and goat anti-mouse IgG $(1 \mathrm{mg} / \mathrm{mL})$ were applied, in turn, to the test lines (T1 to T3) and control line (line C) on the nitrocellulose membrane. The test strip was assembled as shown in Figure 1 and stored in bags with a silica-gel desiccant at $4^{\circ} \mathrm{C}$.

mLAMP-LFD Assay. The 25- $\mu \mathrm{L}$ mLAMP amplicon reaction was mixed with $75 \mu \mathrm{L}$ of running buffer and incubated for $5 \mathrm{~min}$. The mixture was then added dropwise to the sample pad of the LFD. Results were then observed by the naked eye after the device was left to stand for $5 \mathrm{~min}$.

\section{Principle of the mLAMP-LFD Detection}

The protocol outlined in this study combines the rapid detection of mLAMP with visual assessment of the LFD. The principle of visual detection of mLAMP products on an LFD is shown in Figure 1. In the presence of target bacteria, large amounts of biotin- and FITC-, digoxin- and FITC-, or Texas Red- and FITClabeled amplicons are produced under the trigger of specific labeled primers by mLAMP for the target genes. After mixing with the running buffer, the amplicons are added dropwise to the sample pad of the LFD. The mixture moves through the LFD by capillary action and combines with anti-FITC antibody-AuNPs on the conjugate pad to form an amplicon-FITC-antiFITC antibody-AuNP complex. Then, the complexes continue to migrate along the LFD and are sequentially captured by the test lines coated with streptavidin (T1), anti-digoxin antibody (T2), and anti-Texas Red antibody (T3), which is visualized as a red line because of the aggregation of AuNPs. Then, excessive antiFITC antibody-AuNP conjugates continue migrating and are captured to form the other red line (line C) by nonspecific reactions between IgG and anti-FITC antibody on the AuNP surface. When target bacteria are not present in the sample, only line $\mathrm{C}$ is red and no red lines appear on the test lines. For all test results, line $\mathrm{C}$ must be red to demonstrate the validity of LFD detection.

\section{Sensitivity of $m L A M P-L F D$}

To determine the sensitivity of the LAMP-LFD assays, fresh overnight cultures in LB of Salmonella Typhimurium ATCC 14028, C. sakazakii ATCC 29544, and Staph. aureus ATCC 13565 were 10-fold serially diluted with normal saline. One milliliter of each dilution was plated on LB agar for enumeration, and $1 \mathrm{~mL}$ 
from the same dilution was used to extract genomic DNA as described above. Then, $2 \mu \mathrm{L}$ of each genomic DNA sample was used to determine the sensitivity of the mLAMP-LFD and mLAMP-AGE methods. Image analysis software (Quantity One, Bio-Rad Inc., Hercules, CA) was used to estimate the intensity of line signals on the LFD by converting the red bands to gray values (Lee et al., 2015). All reactions were conducted in triplicate for each concentration of bacteria.

\section{Specificity of $m L A M P-L F D$}

The specificity of the mLAMP-AGE and mLAMPLFD detection methods was validated by testing extracted genomic DNA from all 42 bacterial strains (Table 1); each strain was tested in triplicate. At the same time, to verify that the 3 sets of primers did not cross-react with each other (resulting in false-positive results), different types of target genomic DNA were added to the double or triple system (containing 2 or 3 sets of primers) for amplification. All mLAMP products were analyzed using the multiplex LFD assay.

\section{Stability of the LFD}

As an important indicator for evaluating the mLAMPLFD detection method, stability was determined using an accelerated aging test. Using a modified version of the method reported by Peng et al. (2014), LFD were stored in a closed container with a silica gel desiccant at $45^{\circ} \mathrm{C}$, and mLAMP products were detected using these LFD at 0,10, 20, and $30 \mathrm{~d}$ after storage.

\section{Application in Contaminated PIF Samples}

Commercial PIF samples were obtained from local stores in Harbin, China, and determined to be free of Salmonella spp., Cronobacter spp., and Staph. aureus by standard culture methods (ISO, 2017a,b, 1999). Artificially contaminated PIF samples were then pretreated by using the procedure reported by Pan et al. (2018). Briefly, $10 \mathrm{~g}$ of PIF was reconstituted with 90 $\mathrm{mL}$ of buffer peptone water that was preheated to $44^{\circ} \mathrm{C}$, and Salmonella spp., Cronobacter spp., and Staph. aureus were added at concentrations ranging from $10^{9}$ to $10^{-1} \mathrm{cfu} / \mathrm{mL}$. To avoid matrix effects, samples were
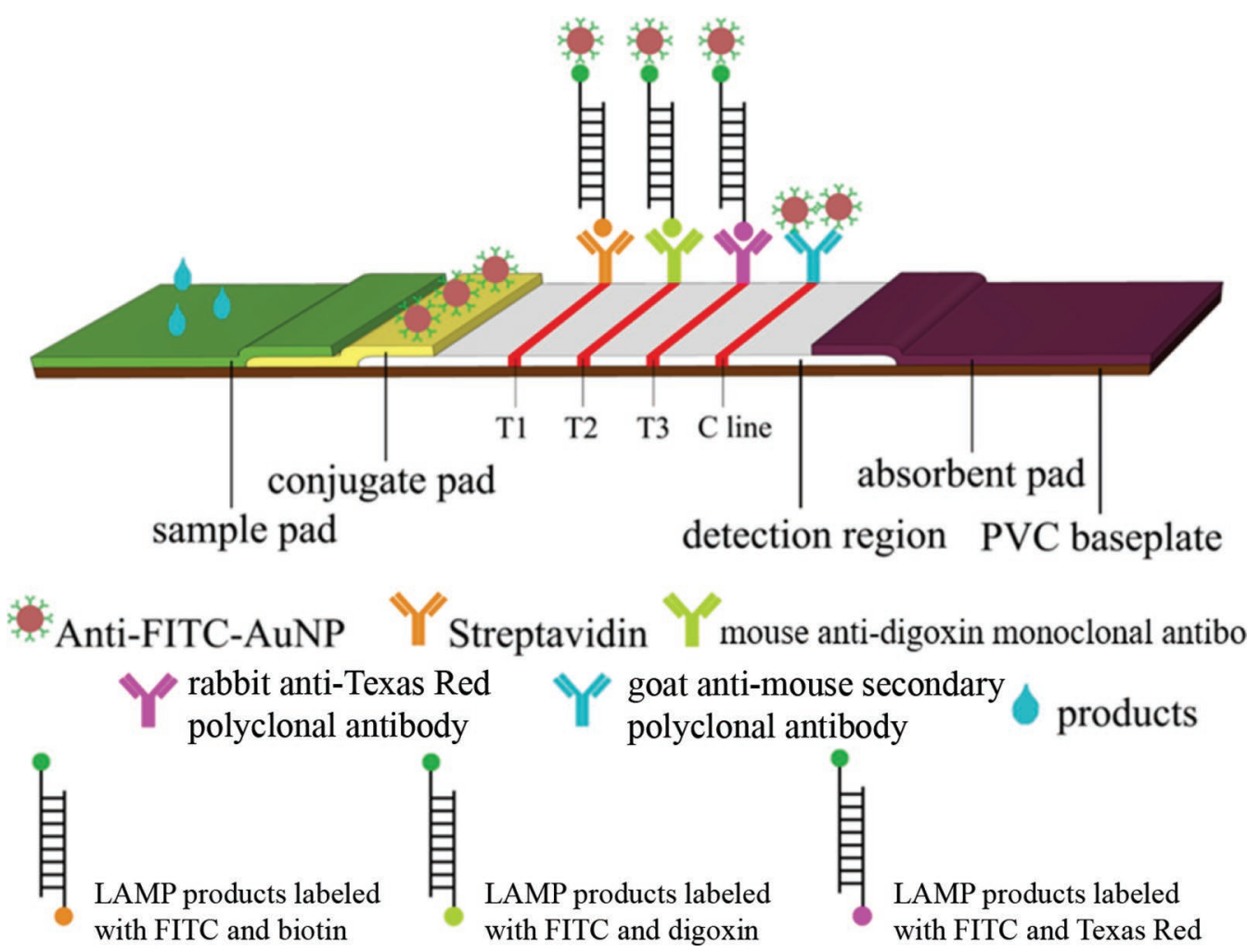

Figure 1. Schematic diagram for the visual detection of multiplex loop-mediated isothermal amplification (mLAMP) products on a lateral flow dipstick (LFD). PVC = polyvinyl chloride; FITC = fluorescein isothiocyanate; AuNP = gold nanoparticle. T1, T2, and T3 are the test lines coated with streptavidin (T1), anti-digoxin antibody (T2), and anti-Texas Red antibody (T3); C is the control line. 
centrifuged for $5 \mathrm{~min}$ at $2,300 \times g$ and $4^{\circ} \mathrm{C}$ to remove the upper layer of whey and fat. Then, the sediment was resuspended in an equal volume of normal saline. Subsequently, $1 \mathrm{~mL}$ of solution was used for extraction of genomic DNA and the mLAMP-LFD assay was conducted as described above. In this paper, detection was assessed by the naked eye, so we did not perform statistical analysis on the results.

\section{RESULTS}

\section{Optimization of the MLAMP-LFD Assay}

To achieve the best detection efficiency and sensitivity, we optimized several key parameters of the mLAMP assay individually, including the concentrations of primers and reagents and reaction temperature and time. Optimal mLAMP conditions were determined from the strongest ladder-like pattern following $2 \%$ AGE. The optimal concentration ratios of F3/B3 to FIP/ BIP (siiA, ITS, nuc) were 1:8, 1:7, and 1:9, respectively (Supplemental File S1 and Figure S1; https://doi.org/ 10.3168/jds.2019-17538). The optimal mLAMP reaction was obtained with $0.9 \mu \mathrm{L}$ of Bst 2.0 WarmStart DNA polymerase, $4 \mathrm{mM} \mathrm{MgSO}_{4}$, and $1.6 \mathrm{~m} M$ dNTP (Supplemental Figure S2; https://doi.org/10.3168/jds .2019-17538) at $64^{\circ} \mathrm{C}$ for 45 min (Supplemental Figure S3; https://doi.org/10.3168/jds.2019-17538). Based on our previous study (Zhao et al., 2017), we further opti- mized the $\mathrm{pH}$ value and $\mathrm{NaCl}$ concentration of the running buffer to achieve sensitive and accurate detection. As shown in Supplemental Figure S4 (https://doi.org/ 10.3168/jds.2019-17538), detection signals on test lines were enhanced at $\mathrm{pH} 9$ compared with that $\mathrm{pH} 7.5$ and $\mathrm{pH}$ 8. However, more false-positive results occurred at $\mathrm{pH}$ 9. To solve this problem, the $\mathrm{NaCl}$ concentration of the running buffer was optimized (Supplemental Figure S4). The color of the test lines decreased with increasing $\mathrm{NaCl}$ concentration. When the $\mathrm{NaCl}$ concentration was $50 \mathrm{~m} M$, the false-positive signal was completely eliminated without affecting detection of positive samples. Thus, the best formula for running buffer was $50 \mathrm{mM}$ $\mathrm{NaCl}$ at $\mathrm{pH} 9$.

As described above, we established the mLAMPLFD detection method and used mLAMP-AGE and mLAMP-LFD to detect different target strains; the results are shown in Figure 2.

\section{Sensitivity of $m L A M P-L F D$}

To determine the sensitivity, genomic DNA preparations of Salmonella spp., Cronobacter spp., and Staph. aureus ranging from $10^{8}$ to $10^{-2} \mathrm{cfu} / \mathrm{mL}$ were detected by mLAMP-LFD and mLAMP-AGE. As shown in Figure 3, weak bands were observed in lane 10 with detection by AGE and LFD. Thus, the limits of detection (LOD) of LFD and AGE are the same; both

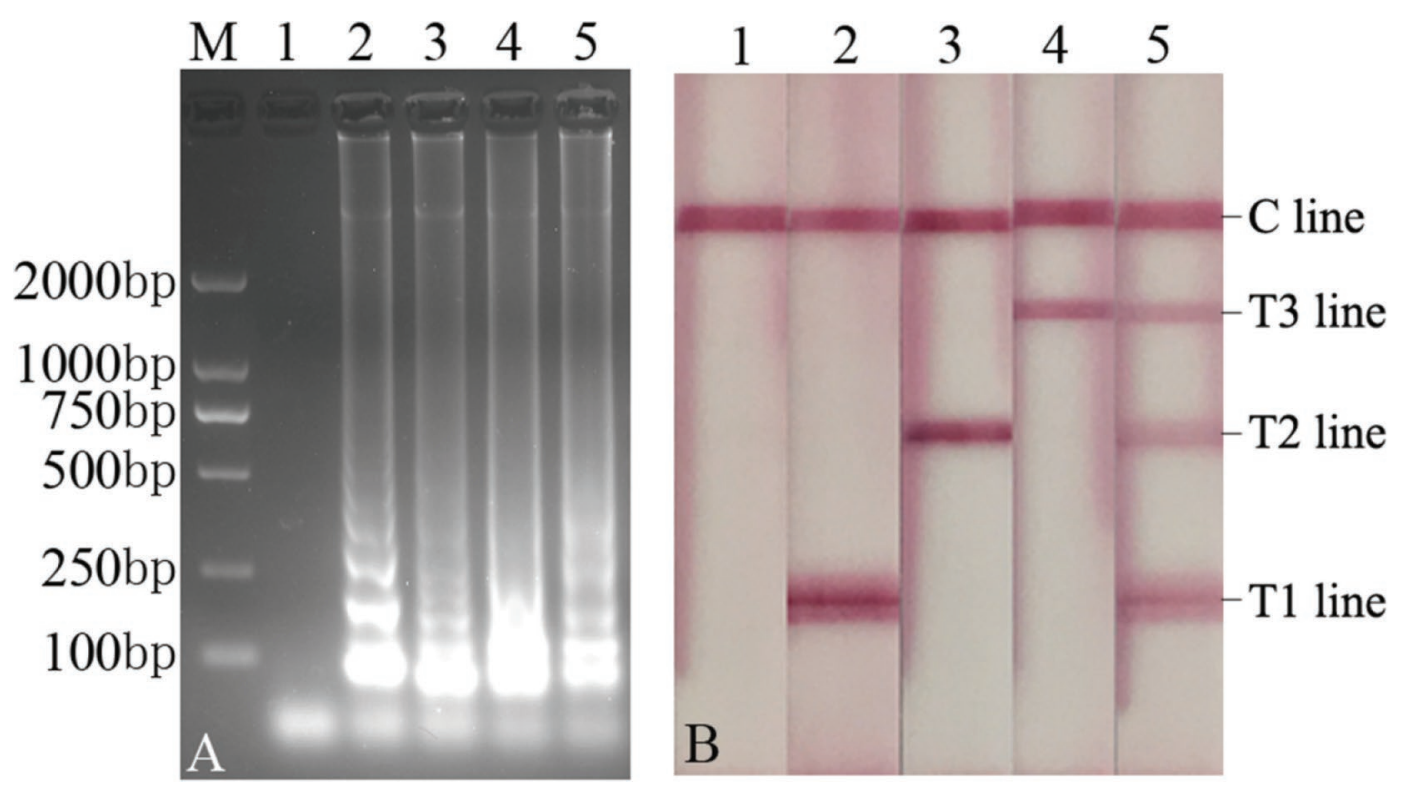

Figure 2. The multiplex loop-mediated isothermal amplification (mLAMP) method with detection of target strains by agarose gel electrophoresis (mLAMP-AGE; A) and by lateral flow dipstick (mLAMP-LFD; B). M = 2,000-bp DNA marker; lane $1=$ negative control; lane $2=$ genomic DNA of Salmonella Typhimurium ATCC 14028; lane $3=$ genomic DNA of Cronobacter sakazakii ATCC 29544; lane $4=$ genomic DNA of Staphylococcus aureus ATCC 13565; lane $5=$ genomic DNA of all 3 bacteria. T1, T2, and T3 are the test lines coated with streptavidin (T1), anti-digoxin antibody (T2), and anti-Texas Red antibody (T3); C is the control line. 
could detect 7, 2.1, and $4.5 \mathrm{cfu} / \mathrm{mL}$ of Salmonella spp., Cronobacter spp., and Staph. aureus, respectively.

For artificially contaminated PIF samples with varying concentrations of 3 target bacteria, the LOD of mLAMP-LFD were consistent with the LOD mLAMPAGE, which were $4.2,2.6$, and $3.4 \mathrm{cfu} / \mathrm{g}$ for Salmonella spp., Cronobacter spp., and Staph. aureus, respectively (Figure 4). However, in Figure 4, only the LFD method

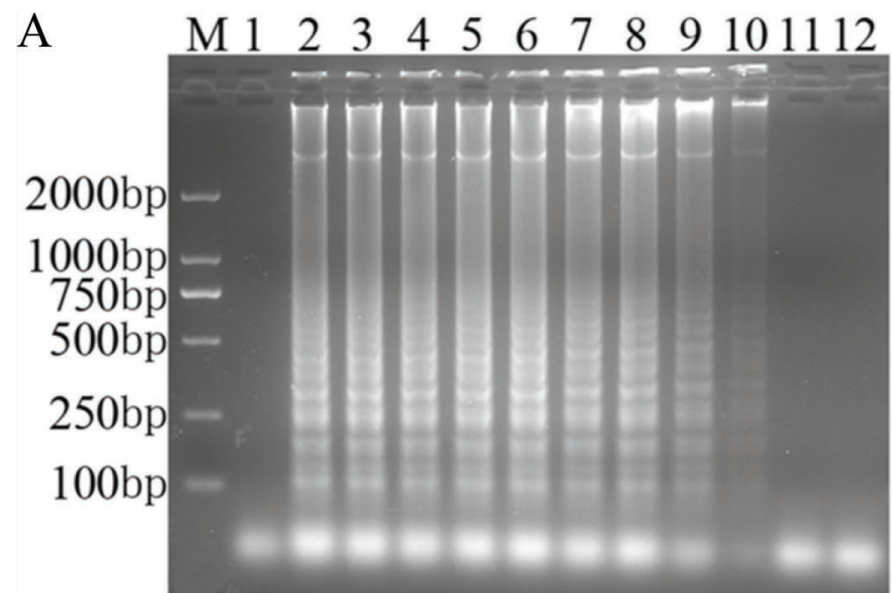

B

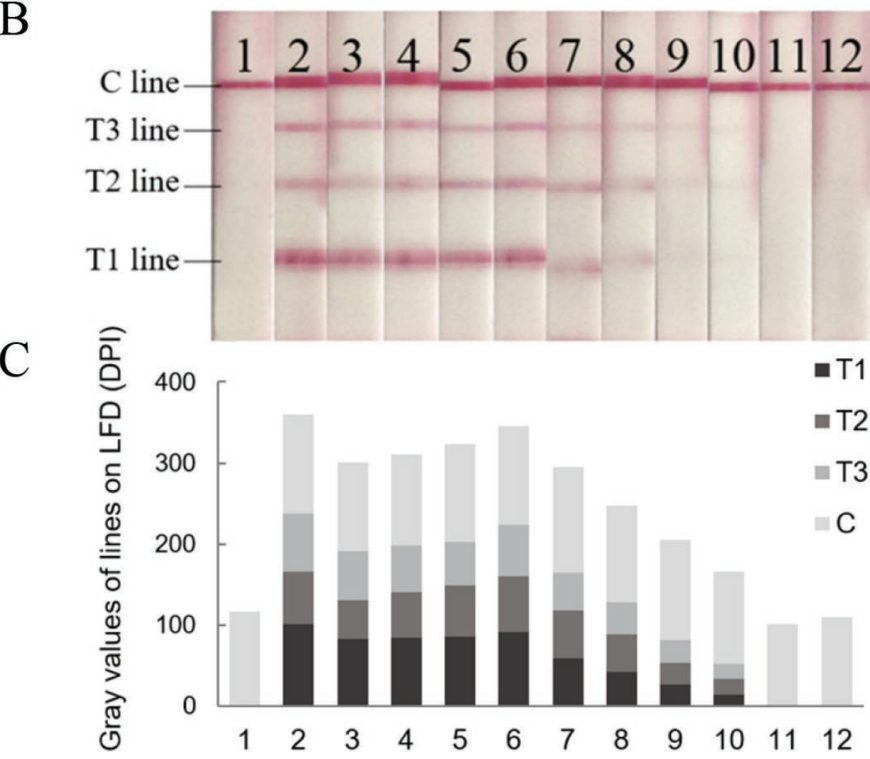

Figure 3. Sensitivity of the multiplex loop-mediated isothermal amplification (mLAMP) methods for pure bacterial culture. (A) Detection of mLAMP products by agarose gel electrophoresis; (B) detection of mLAMP products by lateral flow dipstick (LFD); (C) analysis of gray values of lines on LFD. DPI = dots per inch. $\mathrm{M}=$ 2,000-bp DNA marker; lane $1=$ negative control; lanes 2 to $12=$ genomic DNA of serial concentrations of the Salmonella Typhimurium ATCC 14028, Cronobacter sakazakii ATCC 29544, and Staphylococcus aureus ATCC 13565, ranging from $3.7 \times 10^{8}$ to $3.7 \times 10^{-2} \mathrm{cfu} / \mathrm{mL}$, $2.1 \times 10^{8}$ to $2.1 \times 10^{-2} \mathrm{cfu} / \mathrm{mL}$, and $4.5 \times 10^{8}$ to $4.5 \times 10^{-2} \mathrm{cfu} / \mathrm{mL}$, respectively. T1, T2, and T3 are the test lines coated with streptavidin (T1), anti-digoxin antibody (T2), and anti-Texas Red antibody (T3); $\mathrm{C}$ is the control line.
A

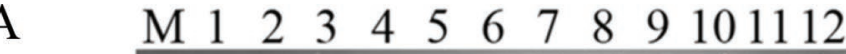

B

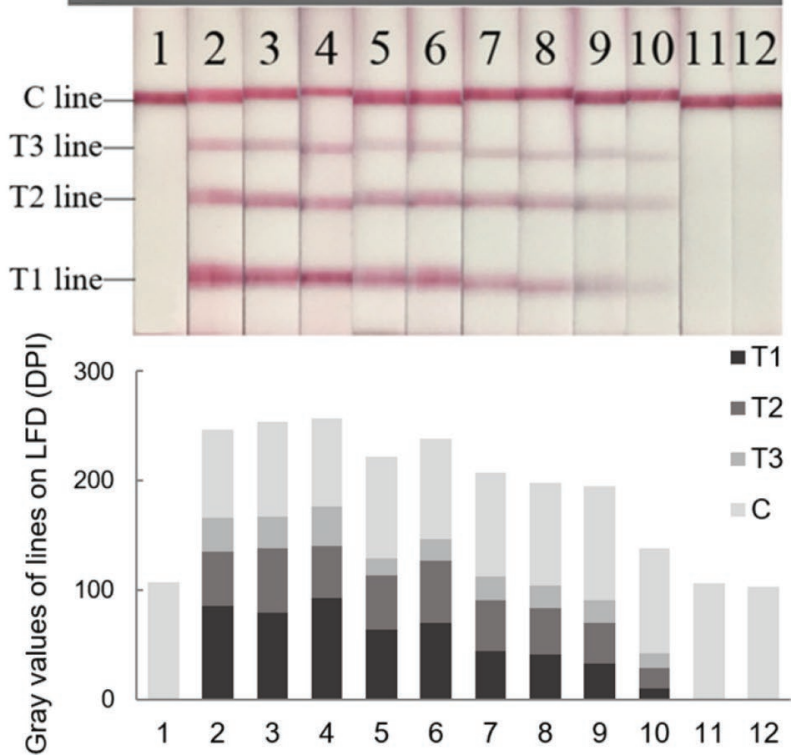

Figure 4. Sensitivity of the multiplex loop-mediated isothermal amplification (mLAMP) methods for powdered infant formula. (A) Detection of mLAMP products by agarose gel electrophoresis; (B) detection of mLAMP products by lateral flow dipstick (LFD); (C) analysis of gray values of lines on LFD. DPI $=$ dots per inch. $\mathrm{M}=2,000-\mathrm{bp}$ DNA marker; lane $1=$ negative control; lanes 2 to $12=$ genomic DNA of serial concentrations of the Salmonella Typhimurium ATCC 14028, Cronobacter sakazakii ATCC 29544, and Staphylococcus aureus ATCC 13565 , ranging from $4.2 \times 10^{8}$ to $4.2 \times 10^{-2} \mathrm{cfu} / \mathrm{g}, 2.6 \times 10^{8}$ to $2.6 \times$ $10^{-2} \mathrm{cfu} / \mathrm{g}$, and $3.4 \times 10^{8}$ to $3.4 \times 10^{-2} \mathrm{cfu} / \mathrm{g}$, respectively. T1, T2, and T3 are the test lines coated with streptavidin (T1), anti-digoxin antibody (T2), and anti-Texas Red antibody (T3); C is the control line.

could clearly distinguish between different species when using multiplex LAMP reactions to produce amplicons.

\section{Specificity of $m L A M P-L F D$}

The mLAMP primers are the main factors affecting the specificity of mLAMP-LFD. Thus, to validate the specificity of the mLAMP-LFD method described here, we considered 2 criteria. First, we validated the 
specificity of the mLAMP method by testing 42 different bacterial strains. The results showed that mLAMPAGE and mLAMP-LFD could detect 13 Salmonella spp., 8 Cronobacter spp., and 6 Staph. aureus strains but did not detect 15 nontarget bacterial strains (Table 1). These results confirmed that the mLAMP-LFD method established here had high specificity for target bacteria. In addition, cross-reactivity was validated by testing the 3 target bacteria with all LAMP primers and without target-specific primers, respectively. The mLAMP reaction without target-specific primers did not detect the corresponding target-specific DNA. Conversely, when all 3 sets of mLAMP primers were included, successful detection of the target-specific DNA was achieved, with no nonspecific detection observed
(Figure 5). These results indicate that the mLAMPLFD method showed no cross-reactivity among primers. Thus, the mLAMP-LFD method can detect and differentiate the 3 target bacteria with high specificity.

\section{Stability of the LFD}

We verified the stability of the prepared LFD by using an accelerated aging test. As shown in Figure 6, for LFD stored at $45^{\circ} \mathrm{C}$ for $30 \mathrm{~d}$, no false-positive results were observed for negative samples and the brightness of the 3 test lines did not change significantly for positive samples. Therefore, storage for $1 \mathrm{mo}$ at $45^{\circ} \mathrm{C}$ did not affect detection performance of LFD, demonstrating stability of the LFD.

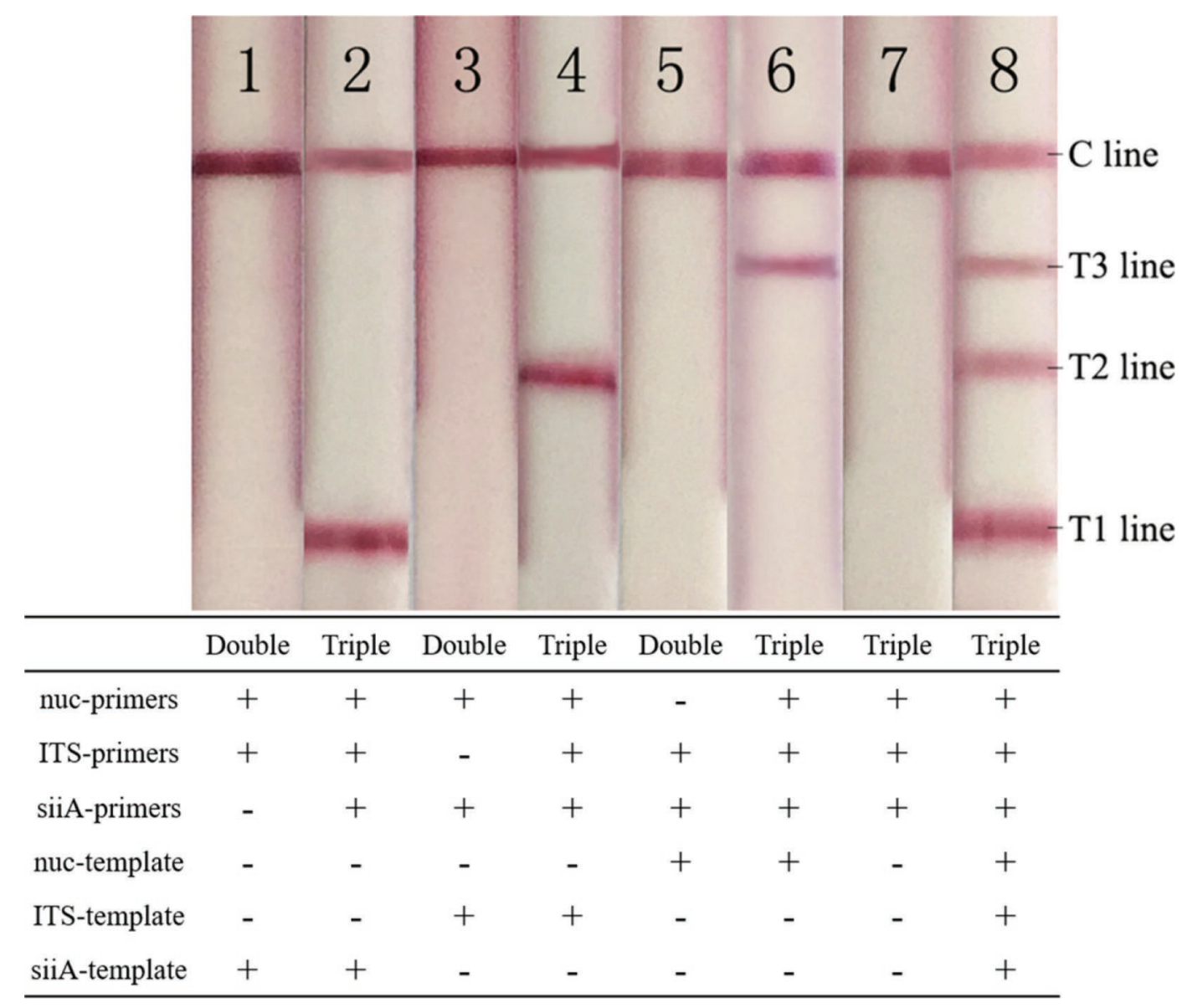

Figure 5. Specificity of the multiplex loop-mediated isothermal amplification (mLAMP)-lateral flow dipstick (LFD) method. Lanes $1,2=$ genomic DNA of Salmonella Typhimurium ATCC 14028 amplified by mLAMP without and with Salmonella target-specific primers, respectively; lanes 3, 4 = genomic DNA of Cronobacter sakazakii ATCC 29544 amplified by mLAMP without and with C. sakazakii target-specific primers, respectively; lanes 5, $6=$ genomic DNA of Staphylococcus aureus ATCC 13565 amplified by mLAMP without and with Staph. aureus target-specific primers, respectively; lanes 7, 8: negative control and template DNA from the 3 target strains detected by mLAMP-LFD. + (-): component present (absent) in mLAMP reaction. T1, T2, and T3 are the test lines coated with streptavidin (T1), anti-digoxin antibody (T2), and anti-Texas Red antibody (T3); C is the control line. 
Table 3. Detection of real samples (artificially contaminated with a certain pathogen) to evaluate the performance of the multiplex loop-mediated isothermal amplification (mLAMP) method in the artificially contaminated powdered infant formula (PIF) samples (values 20 and 40 indicate the number of test samples)

\begin{tabular}{lccccc}
\hline & \multicolumn{4}{c}{ PIF contamination } \\
\cline { 2 - 5 } Method $^{1}$ & Salmonella & Cronobacter & $\begin{array}{c}\text { Staphylococcus } \\
\text { aureus }\end{array}$ & Negative & $\begin{array}{c}\text { Specificity } \\
(\%)\end{array}$ \\
\hline mLAMP-AGE & 20 & 20 & 20 & 40 & 100 \\
mLAMP-LFD & 20 & 20 & 20 & 40 & 100 \\
\hline
\end{tabular}

${ }^{1}$ mLAMP-AGE $=$ detection by mLAMP combined with agarose gel electrophoresis (AGE); mLAMP-LFD = detection by mLAMP combined with lateral flow dipstick (LFD).

\section{Application of mLAMP-LFD Assay in PIF}

To determine the feasibility of the mLAMP-LFD method in PIF samples, we tested 60 positive samples that were artificially contaminated with 1 of 3 different species (20 each for Salmonella spp., Cronobacter spp., and Staph. aureus) and 40 negative samples using the mLAMP-LFD method. All positive samples produced the corresponding test line along with the control line on the LFD. All negative samples only produce the control line of the LFD (Table 3 and Supplemental Figure S5, https://doi.org/10.3168/jds.2019-17538), verifying that the mLAMP-LFD method can be effectively applied to detect 3 different bacterial species in PIF samples.

\section{DISCUSSION}

Salmonella spp., Cronobacter spp., and Staph. aureus are highly prevalent food-borne pathogens globally that cause infant health problems by contaminating PIF

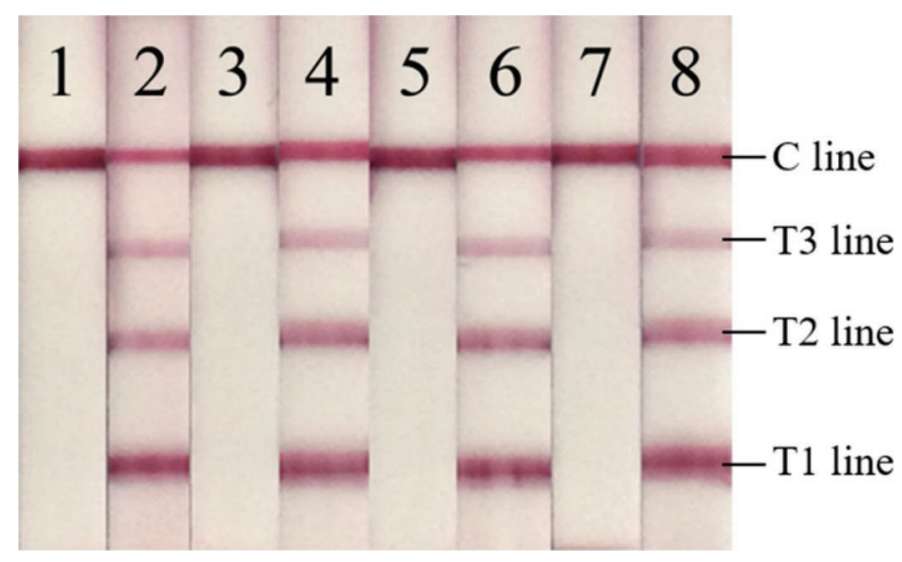

Figure 6. Stability of multiplex loop-mediated isothermal amplification (mLAMP)-lateral flow dipstick (LFD) method. Lanes 1, 3, 5, and $7=\mathrm{LFD}$ stored at $45^{\circ} \mathrm{C}$ for $0,10,20$, and $30 \mathrm{~d}$ and then used to detect mLAMP products of negative control; lanes $2,4,6$, and $8=$ LFD stored at $45^{\circ} \mathrm{C}$ for $0,10,20$, and $30 \mathrm{~d}$ and used to detect mLAMP products of the 3 target strains. T1, T2, and T3 are the test lines coated with streptavidin (T1), anti-digoxin antibody (T2), and antiTexas Red antibody (T3); C is the control line.
(Drudy et al., 2006; Moussa et al., 2010; Wang et al., $2012 \mathrm{~b})$. Because of the low efficiency and high cost of single-pathogen detection, the development of multiple detection systems has great application prospects $(\mathrm{Li}$ and Macdonald, 2016). The LAMP technology has been developed and applied to diverse pathogens because of its high sensitivity and specificity and outstanding efficiency. To date, several target genes have been used in different molecular biology assays for the detection of Salmonella. Among these genes, the siiA gene located on Salmonella pathogenicity island 4 (SPI 4), which plays a crucial role in the survival of macrophages and invasion of polarized cells, has excellent specificity (Gerlach et al., 2008). The internal transcribed spacers (ITS) of 16S-23S rDNA are the most commonly used target sequence in bacterial taxonomy, and the length and sequence vary with different bacterial species. Therefore, the ITS sequence of Cronobacter can be used to design specific primers for the whole genus (Ruan et al., 2013). The nuc gene, which encodes an extracellular enzyme unique to Staph. aureus, was selected because it has been widely used in the molecular detection of the species (Wang et al., 2017).

The development of multiple LAMP-LFD assays is not a simple process and is more challenging than designing single assays. First, to avoid false-negative results, mLAMP must be optimized extensively, including the appropriate quantity of reagents and the concentration ratio for each primer. To determine the optimal primer concentrations, it is necessary to determine the ratio of the outer primer to the inner primer in a single LAMP assay, and then halve it to optimize the double and triple LAMP primers (Shao et al., 2011). Second, the composition of the running buffer affects the accuracy, sensitivity, specificity, and stability of the LFD assay. Currently, PBS (Nurul Najian et al., 2016), Tris (Rivas et al., 2015), saline sodium citrate (Chen et al., 2016), sodium carbonate (Jung et al., 2015), and borate buffer (Peng et al., 2014) have been used as LFD running buffers. Notably, the Tris buffer selected in our study has unique advantages for preventing DNA degradation and dissolution (Rivas et al., 2015). Sucrose and 
trehalose have been used to stabilize AuNPs labeled with antibody conjugates. However, Wang et al. (2013) indicated that trehalose can better maintain conjugate stability than sucrose during storage. Therefore, trehalose was selected in this study to maintain the stability of LFD. Surfactant (Tween-20) has an important role in alleviating nonspecific reactions in the detection zone, inducing better flow of AuNPs on the membrane, and promoting the release of colloidal gold from conjugated mats (Kalogianni et al., 2009; Mao et al., 2009; Hua et al., 2012). Based on these factors, we optimized the composition of the LFD running buffer.

Using the mLAMP-LFD method, the total time for simultaneous detection of Salmonella spp., Cronobacter spp., and Staph. aureus was only $57 \mathrm{~min}$. Furthermore, the results can be monitored clearly and easily in just 10 min (the time to detect the mLAMP products by LFD, including 5 min for incubating products with running buffer and 5 min for LFD standing and reading, which is included in the $57 \mathrm{~min}$ ). Jiang et al. (2012) constructed mLAMP detection systems for simultaneous detection of Shigella, Salmonella, and Staph. aureus in food using AGE to visualize results. However, the AGE method used in that study is time consuming and cannot distinguish the types of amplicons; moreover, the ethidium bromide reagent required in the AGE method is carcinogenic to humans.

During the transportation and storage of LFD, the activity of antigens and antibodies can be affected by environmental factors such as temperature and humidity, resulting in a rapid decrease in the stability of LFD (Peng et al., 2014). In the development process, it is impractical to place the LFD at room temperature for 1 to $2 \mathrm{yr}$ to evaluate its stability. Consequently, to evaluate their stability, we placed the test strips in a high temperature environment for an accelerated aging test. According to the Arrhenius equation (Wang et al., 2009), we estimated that LFD can be stored for more than 1 yr at room temperature or at $4^{\circ} \mathrm{C}$ for more than 2 yr. Thus, the LAMP-LFD method herein developed is rapid, reliable, practical, and stable.

\section{CONCLUSIONS}

The mLAMP-LFD method described here is suitable for rapid, simple, convenient, and sensitive simultaneous detection of Salmonella spp., Cronobacter spp., and Staph. aureus in PIF and dairy food systems that are crucial to the economy of China and many other countries. Compared with conventional mLAMP, our detection method is simple and convenient. At the same time, the method shows high specificity $(100 \%)$ with a detection limit of $<10^{1} \mathrm{cfu} / \mathrm{mL}$ and can be read within $57 \mathrm{~min}$. This method provides a rapid, sensitive, simple, and effective detection system for Salmonella spp., Cronobacter spp., and Staph. aureus in PIF samples and be suitable for point-of-care testing applications in dairy foods.

\section{ACKNOWLEDGMENTS}

This work was supported by the National Natural Science Foundation of China (No. 31871828). The authors declare that they do not have any commercial or associative interest that represents a conflict of interest in connection with the work submitted.

\section{REFERENCES}

Angulo, F. J., S. M. Cahill, I. K. Wachsmuth, M. L. Costarrica, and P. K. B. Embarek. 2008. Powdered infant formula as a source of Salmonella infection in infants. Clin. Infect. Dis. 46:268-273. https: //doi.org/10.1086/524737.

Chen, Y., N. Cheng, Y. Xu, K. Huang, Y. Luo, and W. Xu. 2016. Point-of-care and visual detection of $P$. aeruginosa and its toxin genes by multiple LAMP and lateral flow nucleic acid biosensor. Biosens Bioelectron. 81:317-323. https://doi.org/10.1016/j.bios .2016.03.006.

Dhama, K., K. Karthik, S. Chakraborty, R. Tiwari, S. Kapoor, A. Kumar, and P. Thomas. 2014. Loop-mediated isothermal amplification of DNA (LAMP): A new diagnostic tool lights the world of diagnosis of animal and human pathogens: A review. Pak. J. Biol. Sci. 17:151-166. https://doi.org/10.3923/pjbs.2014.151.166.

Drudy, D., N. R. Mullane, T. Quinn, P. G. Wall, and S. Fanning. 2006. Enterobacter sakazakii: An emerging pathogen in powdered infant formula. Clin. Infect. Dis. 42:996-1002. https://doi.org/10 $.1086 / 501019$.

Gerlach, R. G., N. Cláudio, M. Rohde, D. Jäckel, C. Wagner, and M. Hensel. 2008. Cooperation of Salmonella pathogenicity islands 1 and 4 is required to breach epithelial barriers. Cell. Microbiol. 10:2364-2376. https://doi.org/10.1111/j.1462-5822.2008.01218.x.

He, Y., S. Zhang, X. Zhang, M. Baloda, A. S. Gurung, H. Xu, X. Zhang, and G. Liu. 2011. Ultrasensitive nucleic acid biosensor based on enzyme-gold nanoparticle dual label and lateral flow strip biosensor. Biosens. Bioelectron. 26:2018-2024. https://doi .org/10.1016/j.bios.2010.08.079.

Hua, X., J. Yang, L. Wang, Q. Fang, G. Zhang, and F. Liu. 2012. Development of an enzyme linked immunosorbent assay and an immunochromatographic assay for detection of organophosphorus pesticides in different agricultural products. PLoS One 7:e53099. https://doi.org/10.1371/journal.pone.0053099.

Hyeon, J. Y., C. Park, I. S. Choi, P. S. Holt, and K. H. Seo. 2010. Development of multiplex real-time PCR with internal amplification control for simultaneous detection of Salmonella and Cronobacter in powdered infant formula. Int. J. Food Microbiol. 144:177-181. https://doi.org/10.1016/j.ijfoodmicro.2010.09.022.

ISO. (International Organization for Standardization). 1999. Microbiology of food and animal feeding stuffs - Horizontal method for the enumeration of coagulase-positive staphylococci (Staphylococcus aureus and other species) - Part 1: Technique using Baird-Parker agar medium. ISO standard no. 6888-1:1999. https://www.iso.org/ standard/23036.html.

ISO. (International Organization for Standardization). 2017a. Microbiology of the food chain-Horizontal method for the detection, enumeration and serotyping of Salmonella-Part 1: Detection of Salmonella spp. ISO standard no. 6579-1:2017. https://www.iso .org/standard/56712.html.

ISO. (International Organization for Standardization). 2017b. Microbiology of the Food Chain-Horizontal Method for the Detection of Cronobacter spp. ISO standard no. 22964:2017. https://www.iso .org/standard/64708.html. 
Jiang, K., Q. Lv, D. Zhang, X. Gao, Y. Zhao, M. Li, L. Liu, J. Wu, X. Lu, and C. Luo. 2012. A novel, sensitive, accurate multiplex loopmediated isothermal amplification method for detection of Salmonella spp., Shigella spp. and Staphylococcus aureus in food. J. Food Agric. Environ. 10:252-256. https://doi.org/10.1234/4.2012.3362.

Jung, J. H., S. J. Oh, Y. T. Kim, S. Y. Kim, W.-J. Kim, J. Jung, and T. S. Seo. 2015. Combination of multiplex reverse-transcription loop-mediated isothermal amplification with an immunochromatographic strip for subtyping influenza A virus. Anal. Chim. Acta 853:541-547. https://doi.org/10.1016/j.aca.2014.10.020.

Kalogianni, D. P., I. K. Litos, T. K. Christopoulos, and P. C. Ioannou. 2009. Dipstick-type biosensor for visual detection of DNA with oligonucleotide-decorated colored polystyrene microspheres as reporters. Biosens. Bioelectron. 24:1811-1815. https://doi.org/ 10.1016/j.bios.2008.08.056.

Kokkinos, P. A., P. G. Ziros, M. Bellou, and A. Vantarakis. 2014 Loop-mediated isothermal amplification (LAMP) for the detection of Salmonella in food. Food Anal. Methods 7:512-526. https://doi .org/10.1007/s12161-013-9748-8.

Law, J. W.-F., N.-S. Ab Mutalib, K.-G. Chan, and L.-H. Lee. 2015. Rapid methods for the detection of foodborne bacterial pathogens: Principles, applications, advantages and limitations. Front. Microbiol. 5:770. https://doi.org/10.3389/fmicb.2014.00770.

Lee, J.-H., H. S. Seo, J.-H. Kwon, H.-T. Kim, K. C. Kwon, S. J. Sim, Y. J. Cha, and J. Lee. 2015. Multiplex diagnosis of viral infectious diseases (AIDS, hepatitis C, and hepatitis A) based on point of care lateral flow assay using engineered proteinticles. Biosens. Bioelectron. 69:213-225. https://doi.org/10.1016/j.bios.2015.02.033.

Li, J., and J. Macdonald. 2016. Multiplexed lateral flow biosensors: Technological advances for radically improving point-of-care diagnoses. Biosens. Bioelectron. 83:177-192. https://doi.org/10.1016/ j.bios.2016.04.021.

Liang, C., Y. Chu, S. Cheng, H. Wu, T. Kajiyama, H. Kambara, and G. Zhou. 2012. Multiplex loop-mediated isothermal amplification detection by sequence-based barcodes coupled with nicking endonuclease-mediated pyrosequencing. Anal. Chem. 84:3758-3763. https://doi.org/10.1021/ac3003825.

Liu, J., D. Mazumdar, and Y. Lu. 2006a. A simple and sensitive "dipstick" test in serum based on lateral flow separation of aptamerlinked nanostructures. Angew. Chem. Int. Ed. Engl. 45:7955-7959. https://doi.org/10.1002/anie.200603106.

Liu, Y., X. N. Cai, X. Zhang, Q. L. Gao, X. C. Yang, Z. J. Zheng, M. H. Luo, and X. T. Huang. 2006b. Real time PCR using TaqMan and SYBR Green for detection of Enterobacter sakazakii in infant formula. J. Microbiol. Methods 65:21-31. https://doi.org/10.1016/ j.mimet.2005.06.007.

Mao, X., Y. Q. Ma, A. G. Zhang, L. R. Zhang, L. W. Zeng, and G. D. Liu. 2009. Disposable nucleic acid biosensors based on gold nanoparticle probes and lateral flow strip. Anal. Chem. 81:16601668. https://doi.org/10.1021/ac8024653.

Moussa, I., M. Gassem, A. Al-Doss, W. Sadik, and A. A. Mawgood. 2010. Using molecular techniques for rapid detection of Salmonella serovars in frozen chicken and chicken products collected from Riyadh, Saudi Arabia. Afr. J. Biotechnol. 9:612-619. https://doi .org/10.5897/AJB09.1761.

Nagamine, K., Y. Kuzuhara, T. Notomi, J. Takino, T. Hori, and H. Kanda. 2014. Multi-detection by target mixed loop-mediated isothermal amplification. Int. J. Biochem. Res. Rev. 4:243-252. https: //doi.org/10.9734/IJBCRR/2014/7747.

Notomi, T., H. Okayama, H. Masubuchi, T. Yonekawa, K. Watanabe, N. Amino, and T. Hase. 2000. Loop-mediated isothermal amplification of DNA. Nucleic Acids Res. 28:63e. https://doi.org/10 $.1093 /$ nar/28.12.e63.

Nurul Najian, A. B., E. A. Engku Nur Syafirah, N. Ismail, M. Mohamed, and C. Y. Yean. 2016. Development of multiplex loop mediated isothermal amplification (m-LAMP) label-based gold nanoparticles lateral flow dipstick biosensor for detection of pathogenic Leptospira. Anal. Chim. Acta 903:142-148. https://doi.org/ 10.1016/j.aca.2015.11.015

Pan, R., Y. Jiang, L. Sun, R. Wang, K. Zhuang, Y. Zhao, H. Wang, M. A. Ali, H. Xu, and C. Man. 2018. Gold nanoparticle-based enhanced lateral flow immunoassay for detection of Cronobacter sakazakii in powdered infant formula. J. Dairy Sci. 101:3835-3843. https://doi.org/10.3168/jds.2017-14265.

Peng, T., W. Yang, W.-H. Lai, Y.-H. Xiong, H. Wei, and J. Zhang. 2014. Improvement of the stability of immunochromatographic assay for the quantitative detection of clenbuterol in swine urine. Anal. Methods 6:7394-7398. https://doi.org/10.1039/ C4AY01173B

Rivas, L., A. de la Escosura-Muñiz, L. Serrano, L. Altet, O. Francino, A. Sánchez, and A. Merkoçi. 2015. Triple lines gold nanoparticlebased lateral flow assay for enhanced and simultaneous detection of Leishmania DNA and endogenous control. Nano Res. 8:37043714. https://doi.org/10.1007/s12274-015-0870-3.

Ruan, J., M. Li, Y. P. Liu, Y. Q. Li, and Y. X. Li. 2013. Rapid and sensitive detection of Cronobacter spp. (previously Enterobacter sakazakii) in food by duplex PCR combined with capillary electrophoresis-laser-induced fluorescence detector. J. Chromatogr. B Analyt. Technol. Biomed. Life Sci. 921-922:15-20. https://doi .org/10.1016/j.jchromb.2013.01.008.

Shao, Y., S. Zhu, C. Jin, and F. Chen. 2011. Development of multiplex loop-mediated isothermal amplification-RFLP (mLAMP-RFLP) to detect Salmonella spp. and Shigella spp. in milk. Int. J. Food Microbiol. 148:75-79. https://doi.org/10.1016/j.ijfoodmicro.2011 .05 .004 .

Tanner, N. A., Y. Zhang, and T. C. Evans Jr.. 2012. Simultaneous multiple target detection in real-time loop-mediated isothermal amplification. Biotechniques 53:81-89. https://doi.org/10.2144/ 0000113902

Wang, F., L. Jiang, and B. L. Ge. 2012a. Loop-mediated isothermal amplification assays for detecting Shiga toxin-producing Escherichia coli in ground beef and human stools. J. Clin. Microbiol. 50:91-97. https://doi.org/10.1128/JCM.05612-11.

Wang, X., J. H. Meng, N. Zhang, T. Zhou, Y. M. Zhang, B. W. Yang, M. L. Xi, and X. D. Xia. 2012b. Characterization of Staphylococcus aureus isolated from powdered infant formula milk and infant rice cereal in China. Int. J. Food Microbiol. 153:142-147. https://doi .org/10.1016/j.ijfoodmicro.2011.10.030.

Wang, Y., H. Li, Y. Wang, L. Zhang, J. Xu, and C. Ye. 2017. Loopmediated isothermal amplification label-based gold nanoparticles lateral flow biosensor for detection of Enterococcus faecalis and Staphylococcus aureus. Front. Microbiol. 8:192. https://doi.org/10 .3389 /fcimb.2017.00192.

Wang, Y., H. Xu, M. Wei, H. Gu, Q. Xu, and W. Zhu. 2009. Study of superparamagnetic nanoparticles as labels in the quantitative lateral flow immunoassay. Mater. Sci. Eng. C 29:714-718. https:// doi.org/10.1016/j.msec.2009.01.011.

Wang, Y. K., Y. X. Yan, W. H. Ji, H. A. Wang, S. Q. Li, Q. Zou, and J. H. Sun. 2013. Rapid simultaneous quantification of zearalenone and fumonisin B1 in corn and wheat by lateral flow dual immunoassay. J. Agric. Food Chem. 61:5031-5036. https://doi.org/10 $.1021 /$ jf400803q.

Yang, Y., Q. Li, S. Wang, X. Chen, and A. Du. 2016. Rapid and sensitive detection of Babesia bovis and Babesia bigemina by loopmediated isothermal amplification combined with a lateral flow dipstick. Vet. Parasitol. 219:71-76. https://doi.org/10.1016/j .vetpar.2016.02.004.

Zhao, Y., X. Jiang, Y. Qu, R. Pan, X. Pang, Y. Jiang, and C. Man. 2017. Salmonella detection in powdered dairy products using a novel molecular tool. J. Dairy Sci. 100:3480-3496. https://doi.org/ $10.3168 /$ jds.2016-12535.

\section{ORCIDS}

Yujun Jiang () https://orcid.org/0000-0002-2857-2137 Sihan Chen $\odot$ https://orcid.org/0000-0002-5249-8723 John L. McKillip 나 https://orcid.org/0000-0001-6369-7333

Edward M. Fox ๑ https://orcid.org/0000-0001-5850-7558

Chaoxin Man ำ https://orcid.org/0000-0003-2050-4639 